\title{
Decisão terapêutica na hipertensão: inquérito às atitudes dos médicos de família na região de Lisboa e Vale do Tejo
}

Joana Rodrigues, ${ }^{1}$ Milene Fernandes, ${ }^{2}$ Violeta Alarcão, ${ }^{3}$ Paulo J. Nicola, ${ }^{4}$ Evangelista Rocha ${ }^{5}$

\section{RESUMO}

Objectivos: Caracterizar as atitudes dos médicos de família quanto à decisão terapêutica na hipertensão, incluindo aspectos como controlo e tratamento e comparar estas atitudes com as recomendações clínicas à data do estudo.

Tipo de estudo: Estudo observacional transversal.

Local: Unidades de Cuidados de Saúde Personalizados (UCSP)/Unidades de Saúde Familiar (USF) da região de saúde de Lisboa e Vale do Tejo.

População: Médicos de medicina geral e familiar, especialistas e internos de especialidade.

Métodos: Questionário de auto-preenchimento, com variáveis demográficas e sobre a experiência no seguimento de hipertensos, a medição da pressão arterial (PA), estilos de vida, decisão de início da medicação anti-hipertensora e estratégias usadas na avaliação da adesão à terapêutica. Para identificar associações entre aspectos da decisão e outras variáveis recorreu-se a testes de Qui-Quadrado $(\alpha=0,05)$.

Resultados: Em média, os 60 participantes, dos quais 41 (68,3\%) eram mulheres de 14 UCSP/USF, tinham 52 $\pm 8,6$ anos de idade e 22 $\pm 8,2$ anos de prática clínica. Todos consideraram as modificações de estilo de vida úteis no controlo da PA. Na monitorização 24h da PA (MAPA), os valores diurnos para diagnóstico HTA foram 136/83mmHg. A PA indicada para iniciar tratamento foi, em média, PA>140/90mmHg sem outros factores de risco. Nos idosos, $77 \%$ concorda em aceitar valores de PA mais elevados, iniciando a medicação com PA>150/90mmHg. A monoterapia parece ser preferida no início da terapêutica; $58 \%$ concorda que a maioria dos seus doentes consegue manter a PA controlada com apenas um fármaco e aqueles com mais de 20 anos de prática parecem concordar mais $(p=0,043)$.

Conclusões: As atitudes reportadas estavam alinhadas com as recomendações europeias e nacionais existentes à data, quanto à utilidade das modificações do estilo de vida e os valores considerados para iniciar medicação. Houve alguma heterogeneidade quanto aos valores de PA a considerar no idoso e quanto aos critérios da MAPA. A actualização das recomendações clínicas implica a formação contínua dos médicos para incorporar na prática as melhores opções no seguimento do hipertenso.

Palavras-chave: Hipertensão; Decisão Terapêutica; Recomendações; Relação Médico-Doente.

\section{INTRODUÇÃO}

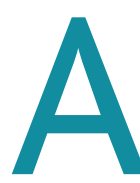
hipertensão arterial (HTA) é um importante problema de saúde pela sua elevada prevalência e por constituir um importante factor de risco para a morbilidade e mortalidade cardiovasculares. ' A nível mundial estima-se que, em 2008, cerca de um bilião de pessoas tivesse HTA, correspon- dendo a aproximadamente $40 \%$ da população com 25 ou mais anos..$^{2-3}$ Em Portugal, dados de 2012 revelaram uma prevalência de HTA de $42,2 \%$ na população adulta, com $74,9 \%$ dos hipertensos sob terapêutica e $55,7 \%$ com a pressão arterial (PA) tratada e controlada. ${ }^{4}$ Uma vez que a relação entre PA e o risco de eventos cardiovasculares é consistente e independente de outros factores de 
risco, o controlo da PA continua a ser uma prioridade. ${ }^{3,5}$

No seguimento de um indivíduo hipertenso, o médico tem de decidir sobre várias opções terapêuticas, de forma a atingir e manter o controlo tensional..$^{6-7}$ Este processo de decisão terapêutica depende de vários factores relacionados com a experiência do médico e características dos doentes e da sua HTA e que podem influenciar a recomendação de modificações do estilo de vida e a decisão de iniciar ou mudar a medicação. ${ }^{8}$ A comunicação entre o médico e o doente é também crucial neste processo, na medida em que os médicos são uma das principais fontes de informação para o doente, mas também porque este possui informação que o médico pode não conhecer, como os valores de PA medidos em casa e problemas de adesão à terapêutica. ${ }^{9}$

O médico de medicina geral e familiar (MGF), pelas características da sua prática clínica, tem um papel muito importante na prevenção, diagnóstico e controlo da HTA. De facto, os motivos de consulta relacionados com doenças do aparelho circulatório têm sido dos mais indicados em consultas nos cuidados de saúde primários, onde o médico de MGF constitui o primeiro e mais acessível contacto. ${ }^{10-11}$ Por outro lado, a maior frequência de contactos e o seguimento ao longo das várias etapas da vida do doente torna possível uma relação de maior confiança que pode facilitar a decisão terapêutica. ${ }^{12-13}$

Este estudo tem, como objectivos, 1) caracterizar as perspectivas dos médicos de MGF quanto à decisão terapêutica na HTA, nomeadamente quanto aos alvos terapêuticos e aspectos relacionados com a terapêutica farmacológica e não-farmacológica, e 2) comparar as atitudes na prática clínica com as recomendações clínicas.

\section{MÉTODOS}

Este é um subestudo do projeto DIMATCH-HTA-De-

'Médica Interna de Medicina Interna, Hospital Pulido Valente - Centro Hospitalar Lisboa Norte

${ }^{2}$ Farmacêutica, MSc Epidemiologia. Investigadora no Instituto de Medicina Preventiva e Saúde Pública - Faculdade de Medicina da Universidade de Lisboa. Medical Writer - Eurotrials, Consultores Científicos

${ }^{3}$ Socióloga. Investigadora no Instituto de Medicina Preventiva e Saúde Pública - Faculdade de Medicina da Universidade de Lisboa

${ }^{4}$ Médico de Medicina Geral e Familiar, MSc Clinical Research. Investigador no Instituto de Medicina Preventiva e Saúde Pública - Faculdade de Medicina da Universidade de Lisboa

${ }^{5}$ Médico Cardiologista, PhD. Coordenador da Unidade de Epidemiologia - Instituto de Medicina Preventiva e Saúde Pública - Faculdade de Medicina da Universidade de Lisboa terminants and Impact of Medication Adherence and Therapeutic Change in the Control of Arterial Hypertension among Cohorts of Immigrants and Non-Immigrants at the Primary Care Level (PTDC/SAU-ESA/ 103511/2008) -, que foi um estudo observacional de coorte prospectiva com amostragem multi-etápica de hipertensos medicados seguidos nos cuidados de saúde primários na região de saúde de Lisboa eVale do Tejo.

Para este subestudo foi considerada a amostra de médicos que aceitaram participar no estudo DIMATCH-HTA, incluindo no estudo piloto, e que foram seleccionados da seguinte forma:

- No estudo piloto foram convidados a participar cinco médicos escolhidos de forma aleatória, de duas Unidades de Saúde Familiar seleccionadas por conveniência.

- No estudo principal, 16 Unidades de Cuidados de Saúde Personalizados (USCP) ou USF foram seleccionadas de forma aleatória e identificados os utentes elegíveis a partir da informação do sistema de informação SINUS: hipertensos medicados, de etnia branca e com naturalidade portuguesa (coorte nativa) ou de etnia negra e de um dos países de língua oficial portuguesa (coorte imigrante), com idade entre 40 e 80 anos e registo de consulta com o médico de família nos últimos 12 meses. Em cada UCSP/USF foram seleccionados, de forma aleatória e convidados para o estudo, cinco médicos de família com pelo menos 40 hipertensos medicados de cada coorte na sua lista de utentes (amostragem por clusters). Nas situações em que a UCSP/USF não tinha cinco médicos com 40 utentes imigrantes elegíveis foram seleccionados os médicos com maior número destes utentes. Caso um médico seleccionado recusasse participar no estudo DIMATCH-HTA era substituído por outro médico seleccionado de igual forma.

A cada médico seleccionado e que consentiu participar no estudo DIMATCH-HTA foi entregue um questionário. O objectivo do estudo foi explicado em detalhe numa sessão de esclarecimento realizada na UCSP/USF e foi obtido o consentimento através do auto-preenchimento do questionário confidencial, sem elementos identificadores.

A elaboração do questionário teve por base as recomendações clínicas para a HTA à data de 2009, ${ }^{14-18}$ além de outros estudos semelhantes. ${ }^{1,19-20} \mathrm{O}$ questionário foi 
pré-testado por elementos da equipa e médicos de medicina geral e familiar. A versão final recolheu informação sobre dados demográficos e de caracterização da experiência clínica no seguimento de doentes hipertensos, quais as fontes de informação consultadas e relacionadas com a HTA, qual a perspectiva face à medição da PA, recomendação de medidas não farmacológicas e decisão de início ou mudança da terapêutica farmacológica anti-hipertensora e quais as estratégias usadas na avaliação da adesão à terapêutica.

Foi realizada análise estatística descritiva, com o cálculo de médias e desvios-padrão (DP) para variáveis quantitativas e frequências relativas para variáveis categóricas. Recorreu-se a análise bivariada (teste quiquadrado) para explorar a relação entre o nível de concordância com diversas afirmações acerca da perspectiva do médico no tratamento da hipertensão e dados demográficos e da prática clínica do médico.

O estudo DIMATCH-HTA foi autorizado pela Comissão de Ética da Faculdade de Medicina de Lisboa, pela Comissão Nacional de Protecção de Dados e pela Administração Regional de Saúde de Lisboa e Vale do Tejo. Os Agrupamentos de Centros de Saúde deram a sua autorização para a colaboração das Unidades de Saúde no estudo.

\section{RESULTADOS}

\section{Caracterização da amostra}

Foram seleccionadas 18 unidades de saúde de nove ACES, das quais cinco unidades de saúde de três ACES recusaram participar por terem como população-alvo apenas uma minoria de imigrantes ou por indisponibilidade logística. Em cada uma das 13 unidades de saúde participantes foram seleccionados e convidados a participar cinco médicos, segundo o método anteriormente descrito. Verificaram-se 20 recusas de participação no estudo DIMATCH-HTA, com a substituição por outros médicos nas mesmas unidades de saúde. Assim, foi obtida uma amostra final de 63 médicos (uma das unidades de saúde apenas tinha três médicos com utentes pertencentes às duas coortes), dos quais três recusaram participar por falta de tempo para preencher o questionário.

Os 60 médicos participantes (41 do sexo feminino) têm 52 $\pm 8,6$ anos (média \pm DP) de idade, licenciaram-se em medicina há $27 \pm 8,2$ anos e têm $22 \pm 8,2$ anos de prá- tica clínica como especialistas em medicina geral e familiar. Em relação a outras actividades, sete $(11,7 \%)$ médicos orientavam ou já tinham orientado estágios, três $(5,0 \%)$ indicaram ter ou ter tido actividade académica, dois $(3,3 \%)$ estavam ou estiveram envolvidos em formação clínica e dois $(3,3 \%)$ exerciam ou tinham exercido actividades de coordenação/direcção de serviços de saúde.

Os médicos participantes indicaram seguir em média $1.782 \pm 176$ doentes, realizar $113 \pm 41,1$ consultas por semana, com uma duração média de 19 $\pm 5,4$ minutos e das quais $23 \pm 22,2$ tinham a HTA como motivo principal.

\section{Perspectivas quanto ao seguimento de doentes hipertensos}

O Quadro I apresenta a distribuição de respostas quanto ao grau de concordância com várias afirmações acerca da HTA e do seguimento clínico desta doença. Todos os médicos concordaram que o controlo da HTA é um problema de saúde importante, que as modificações de estilos de vida são úteis para controlar a PA elevada e que é necessário o uso de fármacos para controlar a PA na maioria dos seus doentes hipertensos.

Quanto à terapêutica farmacológica, 58,3\% dos médicos concordou que a maioria dos seus doentes consegue manter a PA controlada com apenas um anti-hipertensor, tendo sido observado que os médicos com mais de 20 anos de prática clínica em MGF parecem concordar mais com esta afirmação $(80 \%$ vs. $50 \%, p=0,043)$. A maioria concordou que é frequente ter de mudar a medicação devidoà falta de efectividade do(s) anti-hipertensor(es) (94\%) e que é frequente ter de mudar a medicação devido à não-adesão do doente (58\%), sendo que os médicos com idade $>45$ anos ( $75,8 \%$ vs. $27,3 \%, p=0,004)$ e com mais de 20 anos de prática $(75,8 \%$ vs. $20 \%, p<0,001)$ discordaram mais com esta última afirmação.

A totalidade dos médicos concordou que educar os hipertensos quanto à sua doença e ao seu tratamento ajuda no controlo da sua HTA e $98 \%$ concordou que é frequente ensinar os hipertensos a realizar a auto-vigilância dos seus níveis tensionais. Observou-se uma associação entre a concordância com a afirmação ser importante estabelecer consultas regulares com os seus doentes para vigilância da HTA e ter mais de 20 anos desde a licenciatura ( $100 \%$ vs. $83,3 \%, p=0,018)$, apesar de a associação com os anos de prática clínica em MGF 


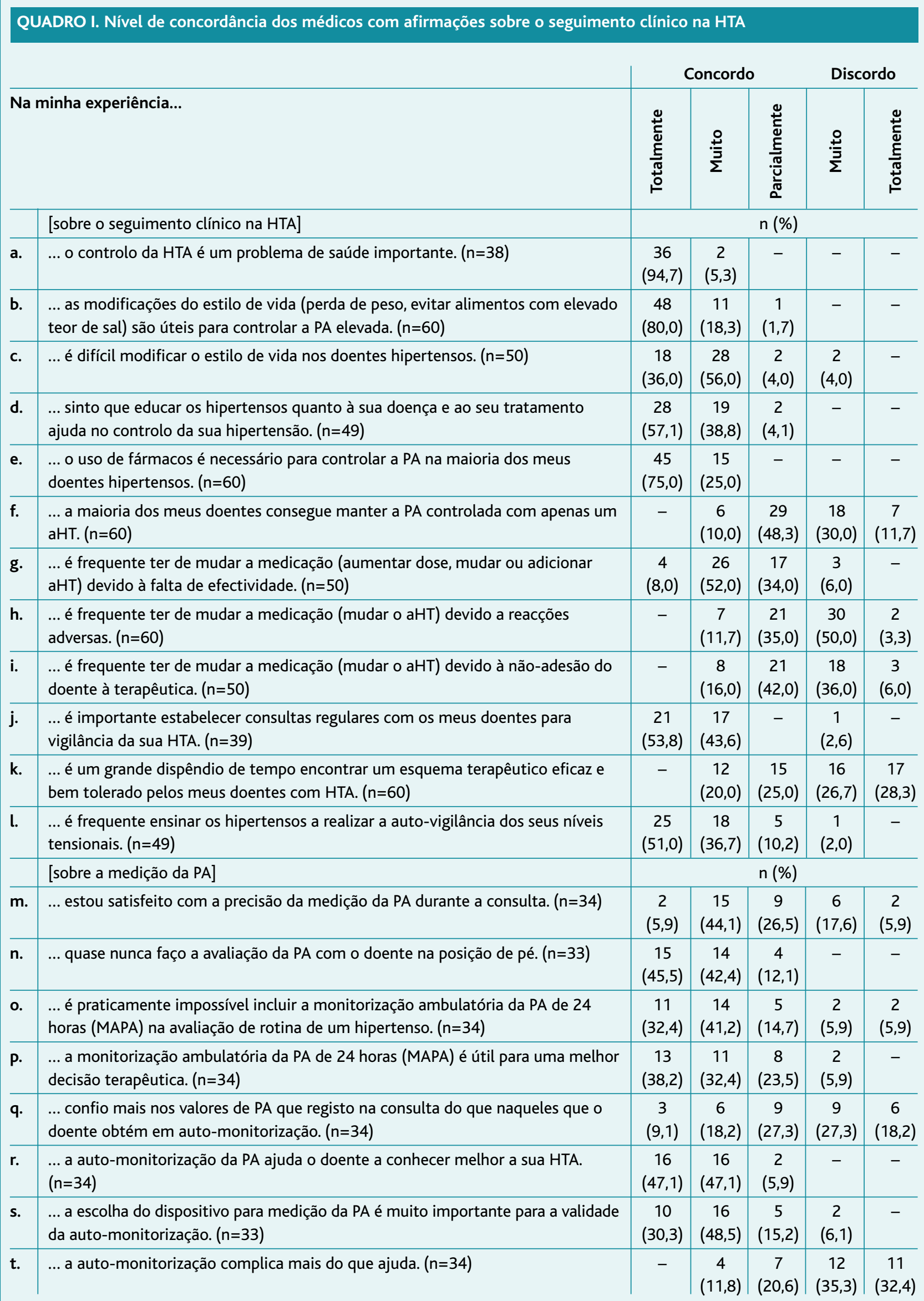




\begin{tabular}{|c|c|c|c|}
\hline \multirow[b]{2}{*}{ 1. Avaliar o conhecimento do doente sobre a sua HTA. } & \multicolumn{2}{|c|}{ Importância ( $\pm D P)$} & \multirow{2}{*}{$\begin{array}{c}\text { \% Consultas } \\
63,6\end{array}$} \\
\hline & 8,0 & $( \pm 2,3)$ & \\
\hline 2. Conhecer os estilos de vida do doente (dieta, tabagismo, ...). & 9,6 & $( \pm 1,3)$ & 87,5 \\
\hline 3. Conversar com o doente sobre a terapêutica actual. & 8,9 & $( \pm 1,9)$ & 70,0 \\
\hline 4. Aferir a capacidade do doente em cumprir/aderir à terapêutica actual. & 9,6 & $( \pm 1,4)$ & 82,1 \\
\hline 5. Medir a PA e outros exames necessários. & 9,9 & $( \pm 1,4)$ & 90,7 \\
\hline 6. Informar o doente do nível de controlo da HTA. & 9,6 & $( \pm 1,3)$ & 86,6 \\
\hline 7. Envolver o doente no processo de decisão terapêutica. & 9,1 & $( \pm 1,9)$ & 73,0 \\
\hline 8. Dar informação ao doente que lhe permita decidir (genéricos). & 7,9 & $( \pm 2,5)$ & 67,5 \\
\hline 9. Explicar os benefícios da medicação. & 9,4 & $( \pm 1,5)$ & 78,4 \\
\hline 10. Explicar quais os efeitos adversos que podem acontecer. & 8,6 & $( \pm 2,0)$ & 61,8 \\
\hline 11. Dar instruções completas sobre a posologia. & 9,9 & $( \pm 0,9)$ & 86,6 \\
\hline 12. Aconselhar a modificação de estilos de vida. & 9,9 & $( \pm 0,5)$ & 88,9 \\
\hline 13. Aconselhar a auto-monitorização da PA. & 9,2 & $( \pm 1,8)$ & 75,5 \\
\hline
\end{tabular}

Nota: 0 - "nada importante"; ... 10 - "muito importante; HTA - Hipertensão arterial; PA - Pressão arterial.

não ter sido observada $(p=0,054)$.

Foi pedido aos médicos que referissem a importância atribuída (numa escala de 0 "nada importante" a 10 "muito importante") a várias componentes da consulta a um hipertenso e que indicassem a percentagem aproximada de consultas em que teriam realizado as mesmas componentes-Quadro II. As componentes da consulta (média \pm DP da pontuação na escala) às quais foi atribuída uma maior importância pelos médicos foram "Medir a PA e outros exames necessários" $(9,9 \pm 1,4)$, "Dar instruções completas sobre a posologia" $(9,9 \pm 0,9) \mathrm{e}$ "Aconselhar a modificação de estilos de vida" $(9,9 \pm 0,5)$. As componentes mais frequentemente indicadas como sendo realizadas na consulta foram "Medir a PA e outros exames necessários" (90,7\%), "Aconselhar a modificação de estilos de vida" (88,9\%) e "Conhecer os estilos de vida do doente (dieta, tabagismo, ...)” (87,5\%).

Perspectivas quanto à medição da $\mathrm{PA}$, em consulta e em auto-monitorização

Conforme indicado no Quadro I, a maioria dos médicos $(88,2 \%)$ concordou que é praticamente impossí- vel incluir a monitorização ambulatória da PA de 24 horas (MAPA) na avaliação de rotina de um hipertenso, apesar de 94,1\% ter concordado que a MAPA é útil para uma melhor decisão terapêutica. Em média, o valor máximo considerado pelos médicos como aceitável para a média de uma PA diurna, medida por MAPA, foi 136 $\pm 8,6 / 83 \pm 4,1 \mathrm{mmHg}$ (máximo indicado de 160/90 e mínimo de $120 / 80 \mathrm{mmHg})$.

Cerca de metade $(54,6 \%)$ dos médicos concordou que confia mais nos valores de PA que regista na consulta do que naqueles que o doente obtém em auto-monitorização, verificando-se uma maior proporção de médicos com 20 ou menos anos de prática em MGF a concordar com esta afirmação ( $64,0 \%$ vs. $14,3 \%, p=0,020)$. A totalidade dos médicos concordou que a auto-monitorização da PA ajuda o doente a conhecer melhor a sua HTA e $94 \%$ concordou que a escolha do dispositivo para medição da PA é muito importante para a validade da auto-monitorização. Para medição da PA na consulta, os médicos indicaram utilizar um esfigmomanómetro aneróide ( $\mathrm{n}=21,63,6 \%)$, um dispositivo electrónico $(n=8,24,2 \%)$ ou estes dois dispositivos $(n=2,6,2 \%)$, sen- 
do menos frequentes o uso do esfigmomanómento de mercúrio, apenas ( $n=1,3,0 \%)$ ou em conjunto com os dispositivos anteriores $(\mathrm{n}=1,3,0 \%)$.

\section{Decisão terapêutica e valores tensionais}

O Quadro III apresenta os valores de pressão arterial considerados na decisão terapêutica quanto ao início de medidas farmacológicas e não farmacológicas e na avaliação do controlo da HTA para diferentes subgrupos de hipertensos.

De uma forma geral, os médicos indicaram recomendar medidas não farmacológicas a um indivíduo sem outros factores de risco cardiovascular, a partir de

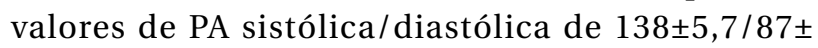
$4 \mathrm{mmHg}$, em média, com 39 (73,1\%) a recomendar para valores de PA sistólica $\geq 140 \mathrm{mmHg}$ e $32(60,4 \%)$ para PA

\begin{tabular}{|c|c|c|c|c|c|c|c|c|}
\hline & \multicolumn{4}{|c|}{$\begin{array}{c}\text { PA sistólica } \\
\text { mmHg (\% respostas)* }\end{array}$} & \multicolumn{4}{|c|}{$\begin{array}{c}\text { PA diastólica } \\
\mathrm{mmHg} \text { (\% respostas)* }\end{array}$} \\
\hline & mín. & $\begin{array}{l}\text { média } \\
\pm D P\end{array}$ & moda & máx. & mín. & $\begin{array}{l}\text { média } \\
\pm \mathrm{DP}\end{array}$ & moda & máx. \\
\hline \multicolumn{9}{|c|}{ Na sua experiência e de uma forma geral, a partir de que valores de PA indica medidas não farmacológicas a um indivíduo... } \\
\hline a. ... sem outros factores de RCV & $\begin{array}{r}120 \\
(1,9)\end{array}$ & $\begin{array}{r}138 \\
\pm 5,7\end{array}$ & $\begin{array}{c}140 \\
(71,2)\end{array}$ & $\begin{array}{r}160 \\
(1,9)\end{array}$ & $\begin{array}{c}80 \\
(17,0)\end{array}$ & $\begin{array}{l}87 \\
\pm 4,0\end{array}$ & $\begin{array}{c}90 \\
(58,5)\end{array}$ & $\begin{array}{c}95 \\
(1,9)\end{array}$ \\
\hline b. ... idoso ( $>75$ anos) sem outros factores de RCV & $\begin{array}{l}120 \\
(3,0)\end{array}$ & $\begin{array}{r}144 \\
\pm 8,2\end{array}$ & $\begin{array}{c}150 \\
(36,4)\end{array}$ & $\begin{array}{c}160 \\
(6,1)\end{array}$ & $\begin{array}{c}80 \\
(15,2)\end{array}$ & $\begin{array}{c}88 \\
\pm 4,6\end{array}$ & $\begin{array}{c}90 \\
(48,5)\end{array}$ & $\begin{array}{c}95 \\
(15,2)\end{array}$ \\
\hline \multicolumn{9}{|c|}{ Na sua experiência e de uma forma geral, a partir de que valores de PA inicia a medicação (anti-hipertensora) a um indivíduo... } \\
\hline c. ... sem outros factores de RCV & $\begin{array}{c}140 \\
(58,8)\end{array}$ & $\begin{array}{r}145 \\
\pm 7,3\end{array}$ & $\begin{array}{c}140 \\
(58,8)\end{array}$ & $\begin{array}{c}160 \\
(14,7)\end{array}$ & $\begin{array}{c}80 \\
(2,9)\end{array}$ & $\begin{array}{c}91 \\
\pm 4,3\end{array}$ & $\begin{array}{c}90 \\
(61,8)\end{array}$ & $\begin{array}{r}100 \\
(8,8)\end{array}$ \\
\hline d. $\quad .$. idoso ( $>75$ anos) sem outros factores de RCV & $\begin{array}{l}130 \\
(2,9)\end{array}$ & $\begin{array}{r}150 \\
\pm 8,2\end{array}$ & $\begin{array}{c}150 \\
(41,2)\end{array}$ & $\begin{array}{c}160 \\
(26,5)\end{array}$ & $\begin{array}{c}80 \\
(5,9)\end{array}$ & $\begin{array}{c}91 \\
\pm 5,2\end{array}$ & $\begin{array}{c}90 \\
(52,9)\end{array}$ & $\begin{array}{c}100 \\
(14,7)\end{array}$ \\
\hline e. ... de etnia negra sem outros factores de RCV & $\begin{array}{l}130 \\
(6,1)\end{array}$ & $\begin{array}{r}143 \\
\pm 7,5\end{array}$ & $\begin{array}{c}140 \\
(57,6)\end{array}$ & $\begin{array}{l}160 \\
(9,1)\end{array}$ & $\begin{array}{c}80 \\
(9,1)\end{array}$ & $\begin{array}{c}90 \\
\pm 5,0\end{array}$ & $\begin{array}{c}90 \\
(51,5)\end{array}$ & $\begin{array}{r}100 \\
(6,1)\end{array}$ \\
\hline f. ... com insuficiência cardíaca & $\begin{array}{r}120 \\
(6,3)\end{array}$ & $\begin{array}{r}136 \\
\pm 6,8\end{array}$ & $\begin{array}{c}140 \\
(46,9)\end{array}$ & $\begin{array}{l}150 \\
(6,3)\end{array}$ & $\begin{array}{c}70 \\
(6,3)\end{array}$ & $\begin{array}{c}85 \\
\pm 6,1\end{array}$ & $\begin{array}{c}90 \\
(37,5)\end{array}$ & $\begin{array}{r}100 \\
(3,1)\end{array}$ \\
\hline g. ... diabético & $\begin{array}{c}120 \\
(8,6)\end{array}$ & $\begin{array}{r}132 \\
\pm 5,8\end{array}$ & $\begin{array}{c}130 \\
(57,1)\end{array}$ & $\begin{array}{l}150 \\
(2,9)\end{array}$ & $\begin{array}{c}70 \\
(5,7)\end{array}$ & $\begin{array}{c}82 \\
\pm 4,9\end{array}$ & $\begin{array}{c}80 \\
(45,7)\end{array}$ & $\begin{array}{c}90 \\
(14,3)\end{array}$ \\
\hline $\begin{array}{l}\text { h. ... idoso (>75 anos) com angina e Diabetes } \\
\text { mellitus }\end{array}$ & $\begin{array}{c}120 \\
(11,8)\end{array}$ & $\begin{array}{r}134 \\
\pm 7,3\end{array}$ & $\begin{array}{c}140 \\
(32,4)\end{array}$ & $\begin{array}{l}150 \\
(2,9)\end{array}$ & $\begin{array}{c}75 \\
(5,9)\end{array}$ & $\begin{array}{c}83 \\
\pm 4,3\end{array}$ & $\begin{array}{c}80 \\
(44,1)\end{array}$ & $\begin{array}{c}90 \\
(17,6)\end{array}$ \\
\hline i. ... com insuficiência renal & $\begin{array}{c}110 \\
(2,9)\end{array}$ & $\begin{array}{r}132 \\
\pm 9,2\end{array}$ & $\begin{array}{c}130 \\
(35,3)\end{array}$ & $\begin{array}{l}160 \\
(2,9)\end{array}$ & $\begin{array}{c}70 \\
(5,9)\end{array}$ & $\begin{array}{c}82 \\
\pm 5,3\end{array}$ & $\begin{array}{c}80 \\
(55,9)\end{array}$ & $\begin{array}{c}90 \\
(20,6)\end{array}$ \\
\hline \multicolumn{9}{|c|}{ Quais os valores de PA que, na sua experiência, definem um doente com hipertensão controlada? } \\
\hline j. .... com RCV ligeiro a moderado & $\begin{array}{c}120 \\
(1,9)\end{array}$ & $\begin{array}{r}136 \\
\pm 5,3\end{array}$ & $\begin{array}{c}140 \\
(53,8)\end{array}$ & $\begin{array}{c}145 \\
(1,9)\end{array}$ & $\begin{array}{c}70 \\
(3,6)\end{array}$ & $\begin{array}{c}84 \\
\pm 5,4\end{array}$ & $\begin{array}{c}80 \\
(41,8)\end{array}$ & $\begin{array}{c}95 \\
(1,8)\end{array}$ \\
\hline k. ... com RCV elevado & $\begin{array}{l}100 \\
(1,9)\end{array}$ & $\begin{array}{r}128 \\
\pm 7,0\end{array}$ & $\begin{array}{c}130 \\
(61,1)\end{array}$ & $\begin{array}{l}150 \\
(1,9)\end{array}$ & $\begin{array}{c}60 \\
(1,8)\end{array}$ & $\begin{array}{c}79 \\
\pm 6,0\end{array}$ & $\begin{array}{c}80 \\
(49,1)\end{array}$ & $\begin{array}{c}90 \\
(7,3)\end{array}$ \\
\hline
\end{tabular}

Nota: *indicada a \% respostas excepto para média đdesvio-padrão; mín - mínimo; máx - máximo; RCV - Risco cardiovascular; PA - Pressão arterial; HTA - Hipertensão; DP - Desvio-padrão. 
diastólica $\geq 90 \mathrm{mmHg}$. As medidas não farmacológicas mais indicadas como preferidas numa primeira abordagem foram: exercício físico regular $(\mathrm{n}=54,90,0 \%)$; redução do peso ( $n=52,86,7 \%)$; e cessação tabágica $(n=52$, $86,7 \%)$. Relativamente à redução do peso, os médicos mencionaram recomendar esta medida a partir de um Índice de Massa Corporal superior a $26 \pm 4,4 \mathrm{~kg} / \mathrm{m}^{2}$ (média \pm DP). Outras medidas não farmacológicas também indicadas foram o controlo da dislipidémia por dieta $(\mathrm{n}=46,76,7 \%)$, a restrição de sal $(\mathrm{n}=45,75,0 \%)$ e a redução do consumo de álcool ( $\mathrm{n}=33,55,0 \%)$.

Quanto à decisão de iniciar a terapêutica farmacológica (Quadro III) em indivíduos sem outros factores de risco cardiovascular, a PA sistólica/diastólica indicada pelos médicos foi de $145 \pm 7,3 / 91 \pm 4,3 \mathrm{mmHg}$ (média $\pm D P)$, com $10(29,4 \%)$ médicos a indicarem iniciar tratamento quando PA sistólica $\geq 150 \mathrm{mmHg}$ e 8 (23,5\%) quando PA diastólica $\geq 95 \mathrm{mmHg}$. Na presença de outros factores de risco (Diabetes mellitus, insuficiência cardíaca, insuficiência renal, etc.) foram indicados valores tensionais mais baixos.

Quando questionados sobre quais os valores considerados para uma HTA controlada, a PA sistólica/diastólica foi, em média, de 136/84mmHg em doentes com risco cardiovascular ligeiro a moderado e de $128 / 79 \mathrm{mmHg}$ em doentes com risco elevado. Na análise por níveis de PA, 29 (53,7\%) médicos indicaram valores de PA sistólica entre 140 e $145 \mathrm{mmHg}$, inclusive, e $19(34,5 \%)$ indicaram valores de PA diastólica entre 90 e 95mmHg para os indivíduos com risco ligeiro a moderado. Nos doentes com risco elevado, estas proporções diminuíram: três (5,5\%) indicaram valores de PA sistólica entre 140 e $145 \mathrm{mmHg}$, inclusive, e quatro $(7,3 \%)$ valores de PA diastólica de 90mmHg.

\section{Decisão terapêutica no início da medicação anti-hipertensora}

Perante um indivíduo hipertenso, sem outros factores de risco cardiovascular ou na presença de outras características ou co-morbilidades, todos os 34 médicos que responderam a esta questão indicaram preferir iniciar a medicação anti-hipertensora com opções em monoterapia. O Quadro IV apresenta os grupos farmacológicos e associações preferenciais no início da medicação nos diferentes subgrupos. Num indivíduo sem outros factores de risco, a opção mais indicada foi a monoterapia com IECAs (30,9\% em 55 respostas).

No início da medicação anti-hipertensora, a maioria dos médicos (98\%) indicou recomendar a medição da PA em auto-monitorização com diferentes frequências: todos os dias $(n=3,6,5 \%)$, a cada $2-3$ dias $(n=24$, $52,2 \%)$, a cada $1-2$ semanas ( $n=12,26,1 \%)$, uma vez por mês ( $n=2,4,3 \%)$ ou com outras frequências ( $n=4,8,7 \%$ ), enquanto um $(2,2 \%)$ médico indicou não recomendar. Adicionalmente, os médicos indicaram recomendar uma nova consulta médica com diferente periodicidade: até 2 semanas $(n=7,17,5 \%)$; uma vez por mês $(n=13$, $32,5 \%)$; a cada $2-3$ meses $(n=12,30,0 \%)$; ou outra periodicidade $(n=8,13,3 \%)$.

\section{Perspectivas quanto à decisão terapêutica na HTA}

Em média, os médicos consideraram que, tendo em conta o último ano, $7 \%$ dos seus doentes hipertensos se encontravam controlados apenas com medidas não farmacológicas, $27 \%$ controlados em monoterapia, $55 \%$ controlados com mais que um anti-hipertensor e 10\% não controlados com mais que três anti-hipertensores (HTA resistente).

O Quadro V apresenta o nível de concordância dos médicos com afirmações relativas à decisão terapêutica na HTA. A maioria dos médicos discordou que $o$ tratamento da HTA provoca mais problemas do que resolve (94\%) e que a decisão por uma intervenção farmacológica ou não farmacológica varia com o sexo do doente ou (...) com a etnia do doente $(60 \%$ e $51 \%$, respectivamente). Quanto ao idoso hipertenso, $85 \%$ dos médicos concordou que a mudança de estilos de vida é mais difícil nos idosos e $77 \%$ concordou que, em termos de controlo da HTA, aceita valores de PA mais elevados.

A maioria dos médicos considerou mudar frequentemente a terapêutica anti-hipertensora (54\%). Quando questionados sobre os motivos mais importantes para não mudar ou intensificar a terapêutica se a PA não estiver controlada numa dada consulta (Quadro VI), os mais escolhidos pelos médicos foram [o doente] "não tem tomado a medicação como indicado, omite tomas ou deixou terminar a medicação" (23\%), "precisa de ser avaliado numa segunda consulta: esta foi a primeira em que a PA está aumentada" (23\%) e "tem os valores de PA em auto-monitorização normais" (20\%).

O Quadro VII apresenta os factores considerados pelos médicos como associados à não adesão do doente 
QUADRO IV. Fármacos anti-hipertensores preferidos pelos médicos numa primeira abordagem a um hipertenso... (mais do que uma opção possível) $(n=34)$
a) ...sem outros factores de risco cardiovascular
e) ...com insuficiência cardíaca
d) ...idoso (>75 anos) sem outros factores de RCV
f) ...diabético
c) ...de etnia negra sem outros factores de RCV
g) ...com insuficiência renal

d) ...com bronquite crónica ou DPOC

\begin{tabular}{|c|c|c|c|c|c|c|c|}
\hline & a) & b) & c) & d) & e) & f) & g) \\
\hline Total de respostas, n (\%) & $55(100)$ & $64(100)$ & $52(100)$ & $54(100)$ & $66(100)$ & $61(100)$ & $55(100)$ \\
\hline$(*)$ IECA & $16(29,1)$ & $4(6,3)$ & $9(17,3)$ & $18(33,3)$ & $13(19,7)$ & $25(41,0)$ & $17(30,9)$ \\
\hline$(*)$ ARA & $11(20,0)$ & $8(12,5)$ & $12(23,1)$ & $18(33,3)$ & $11(16,7)$ & $24(39,3)$ & $21(38,2)$ \\
\hline$(*) \mathrm{BB}$ & $7(12,7)$ & $2(3,1)$ & - & - & $16(24,2)$ & $1(1,6)$ & $2(3,6)$ \\
\hline$\left(^{*}\right) \mathrm{ACC}$ & $2(3,6)$ & $28(43,8)$ & $8(15,4)$ & $6(11,1)$ & $3(4,5)$ & $6(9,8)$ & $2(3,6)$ \\
\hline$(*)$ TIAZ & $7(12,7)$ & $11(17,2)$ & $12(23,1)$ & $6(11,1)$ & $8(12,1)$ & $2(3,3)$ & $4(7,3)$ \\
\hline$(*) n T I A Z$ & $4(7,3)$ & $4(6,3)$ & $6(11,5)$ & $1(1,9)$ & $8(12,1)$ & $1(1,6)$ & $5(9,1)$ \\
\hline$(*)($ IECA ou ARA $)+$ TIAZ & $5(9,1)$ & $1(1,6)$ & $2(3,8)$ & $2(3,7)$ & - & - & $2(3,6)$ \\
\hline$\left(^{*}\right)($ IECA ou ARA) + outro fármaco & $3(5,5)$ & $2(3,1)$ & $1(1,9)$ & $3(5,6)$ & $4(6,1)$ & $2(3,3)$ & $2(3,6)$ \\
\hline$(*) A C C+$ TIAZ & - & $3(4,7)$ & $1(1,9)$ & - & - & - & - \\
\hline$(*)$ outra associação & - & $1(1,6)$ & $1(1,9)$ & - & $3(4,5)$ & - & - \\
\hline
\end{tabular}

Nota: $\left({ }^{*}\right)$ \% para cada opção apresentada em função do total de respostas; indicadas a negrito as opções mais frequentes. AHs - Anti-hipertensores; IECA - Inibidores da enzima de conversão da angiotensina;ARA - Antagonistas do receptor 1 da angiotensina; BB - Bloqueadores beta; ACC - Antagonistas dos canais de cálcio; TIAZ - Diuréticos tiazídicos; nTIAZ - Outros diuréticos não-tiazídicos; onde indicado "-" considerar "0 (0,0\%)"

à terapêutica. Os mais indicados foram "custos da medicação" (33\%), "desinteresse sobre a sua HTA" (22\%) e "polimedicação associada a co-morbilidades"(18\%).Em média, os médicos consideraram que $68 \pm 17 \%$ dos seus doentes eram aderentes à terapêutica farmacológica, que $78 \pm 17 \%$ cumpriam a data das consultas para seguimento da HTA, $40 \pm 22 \%$ faziam uma dieta com restrição de sal, $51 \pm 24 \%$ consumiam bebidas alcoólicas sem ser de forma excessiva e que $19 \pm 11 \%$ tinham actividade física regular.

\section{DISCUSSÃO}

Neste estudo, as atitudes reportadas pelos médicos estavam alinhadas com as recomendações europeias e nacionais existentes à data, quanto à utilidade das modificações do estilo de vida e os valores considerados para iniciar medicação. Contudo, observou-se alguma heterogeneidade de atitudes face aos valores alvo no tratamento do idoso hipertenso, bem como em relação aos valores de MAPA a considerar como critérios de diagnóstico.

A consulta médica, como momento de interacção entre o médico de família e o indivíduo hipertenso, é um momento fundamental para alcançar um melhor controlo da PA. ${ }^{16}$ No presente estudo, os médicos indicaram realizar mais frequentemente as componentes da consulta que também consideraram mais importantes, nomeadamente medir a PA, pedir outros exames necessários e aconselhar a modificação de estilos de vida.

A totalidade dos médicos concordou que educar os hipertensos quanto à sua doença e ao seu tratamento ajuda no controlo da sua HTA. Contudo, à semelhança de resultados obtidos em estudos anteriores, ${ }^{9}$ verificou- 


\begin{tabular}{|c|c|c|c|c|c|c|}
\hline & & \multicolumn{3}{|c|}{ Concordo } & \multicolumn{2}{|c|}{ Discordo } \\
\hline \multicolumn{2}{|c|}{ Na minha experiência... } & & & \pm & & f \\
\hline & [sobre a decisão terapêutica na HTA] & \multicolumn{5}{|c|}{$\mathrm{n}(\%)$} \\
\hline a. & $\begin{array}{l}\text {... sinto-me relutante em iniciar o tratamento farmacológico num doente com } \\
\text { HTA ligeira e que continua a fumar. }\end{array}$ & - & $\begin{array}{c}2 \\
(5,7)\end{array}$ & $\begin{array}{c}13 \\
(37,1)\end{array}$ & $\begin{array}{c}8 \\
(22,9)\end{array}$ & $\begin{array}{c}12 \\
(34,3)\end{array}$ \\
\hline b. & $\begin{array}{l}\text {... acho que todos os fármacos aHT são semelhantes na sua eficácia no controlo } \\
\text { da PA. }\end{array}$ & $\begin{array}{c}2 \\
(5,7)\end{array}$ & $\begin{array}{c}3 \\
(8,6)\end{array}$ & $\begin{array}{c}10 \\
(28,6)\end{array}$ & $\begin{array}{c}5 \\
(14,3)\end{array}$ & $\begin{array}{c}15 \\
(42,9)\end{array}$ \\
\hline c. & ... acho que o uso de Tiazidas para reduzir a PA está ultrapassado. & - & $\begin{array}{c}2 \\
(5,7)\end{array}$ & $\begin{array}{c}9 \\
(25,7)\end{array}$ & $\begin{array}{c}13 \\
(37,1)\end{array}$ & $\begin{array}{c}11 \\
(31,4)\end{array}$ \\
\hline d. & $\begin{array}{l}\text {... com tudo o que temos para fazer, é impraticável diagnosticar e tratar } \\
\text { adequadamente todos os hipertensos. }\end{array}$ & $\begin{array}{c}2 \\
(5,7)\end{array}$ & $\begin{array}{c}2 \\
(5,7)\end{array}$ & $\begin{array}{c}9 \\
(25,7)\end{array}$ & $\begin{array}{c}7 \\
(20,0)\end{array}$ & $\begin{array}{c}15 \\
(42,9)\end{array}$ \\
\hline e. & ... acho que o tratamento da HTA provoca mais problemas do que resolve. & - & - & $\begin{array}{c}2 \\
(5,7)\end{array}$ & $\begin{array}{c}9 \\
(25,7)\end{array}$ & $\begin{array}{c}24 \\
(68,6)\end{array}$ \\
\hline f. & $\begin{array}{l}\text {... a decisão por uma intervenção farmacológica ou não farmacológica varia com } \\
\text { o sexo do doente. }\end{array}$ & $\begin{array}{c}3 \\
(8,6)\end{array}$ & $\begin{array}{c}1 \\
(2,9)\end{array}$ & $\begin{array}{c}10 \\
(28,6)\end{array}$ & $\begin{array}{c}8 \\
(22,9)\end{array}$ & $\begin{array}{c}13 \\
(37,1)\end{array}$ \\
\hline \multirow[t]{2}{*}{ g. } & $\begin{array}{l}\text {... a decisão por uma intervenção farmacológica ou não farmacológica varia com } \\
\text { a etnia do doente. }\end{array}$ & $\begin{array}{c}7 \\
(20,0)\end{array}$ & $\begin{array}{c}4 \\
(11,4)\end{array}$ & $\begin{array}{c}6 \\
(17,1)\end{array}$ & $\begin{array}{c}7 \\
(20,0)\end{array}$ & $\begin{array}{c}11 \\
(31,4)\end{array}$ \\
\hline & [sobre a decisão terapêutica no idoso com HTA] & \multicolumn{5}{|c|}{$\mathrm{n}(\%)$} \\
\hline h. & $\begin{array}{l}\text {... sinto-me relutante em iniciar um aHT num idoso devido aos possíveis efeitos } \\
\text { secundários. }\end{array}$ & $\begin{array}{c}2 \\
(5,7)\end{array}$ & $\begin{array}{c}2 \\
(5,7)\end{array}$ & $\begin{array}{c}7 \\
(20,0)\end{array}$ & $\begin{array}{c}16 \\
(45,7)\end{array}$ & $\begin{array}{c}8 \\
(22,9)\end{array}$ \\
\hline i. & $\begin{array}{l}\text {... apercebo-me que há mais idosos do que indivíduos mais jovens a ter de } \\
\text { descontinuar a terapêutica devido aos efeitos secundários. }(n=34)\end{array}$ & $\begin{array}{c}1 \\
(2,9)\end{array}$ & $\begin{array}{c}8 \\
(23,5)\end{array}$ & $\begin{array}{c}11 \\
(32,4)\end{array}$ & $\begin{array}{c}9 \\
(26,5)\end{array}$ & $\begin{array}{c}5 \\
(14,7)\end{array}$ \\
\hline j. & $\begin{array}{l}\text {... questiono-me sobre o benefício de começar o tratamento para a HTA em } \\
\text { idosos a partir dos } 75 \text { anos. }\end{array}$ & $\begin{array}{c}1 \\
(2,9)\end{array}$ & $\begin{array}{c}4 \\
(11,4)\end{array}$ & $\begin{array}{c}9 \\
(25,7)\end{array}$ & $\begin{array}{c}16 \\
(45,7)\end{array}$ & $\begin{array}{c}5 \\
(14,3)\end{array}$ \\
\hline k. & ... em termos de controlo da HTA, aceito valores de PA mais elevados nos idosos. & $\begin{array}{c}5 \\
(14,3)\end{array}$ & $\begin{array}{c}14 \\
(40,0)\end{array}$ & $\begin{array}{c}8 \\
(22,9)\end{array}$ & $\begin{array}{c}6 \\
(17,1)\end{array}$ & $\begin{array}{c}2 \\
(5,7)\end{array}$ \\
\hline l. & $\begin{array}{l}\text {... a adesão à terapêutica anti-hipertensora é um problema mais relevante nos } \\
\text { idosos. }(n=34)\end{array}$ & $\begin{array}{c}1 \\
(2,9)\end{array}$ & $\begin{array}{c}9 \\
(26,5)\end{array}$ & $\begin{array}{c}7 \\
(20,6)\end{array}$ & $\begin{array}{c}12 \\
(35,3)\end{array}$ & $\begin{array}{c}5 \\
(14,7)\end{array}$ \\
\hline m. & ... a mudança de estilos de vida é mais difícil nos idosos. $(n=34)$ & $\begin{array}{c}9 \\
(26,5)\end{array}$ & $\begin{array}{c}16 \\
(47,1)\end{array}$ & $\begin{array}{c}4 \\
(11,8)\end{array}$ & $\begin{array}{c}4 \\
(11,8)\end{array}$ & $\begin{array}{c}1 \\
(2,9)\end{array}$ \\
\hline
\end{tabular}

Nota: HTA - Hipertensão; PA - Pressão arterial; aHT - Anti-hipertensor; onde indicado "-" considerar "0 (0,0\%)"

-se que várias componentes da consulta relacionadas com a educação do utente foram consideradas menos importantes e parecem ser menos realizadas, como avaliar o conhecimento do doente sobre a sua HTA, ex- plicar os benefícios e os efeitos adversos da medicação, aferir a capacidade do doente em cumprir/aderir à terapêutica actual ou mesmo envolver o doente no processo de decisão terapêutica. Para aumentar as com- 


\section{QUADRO VI. Motivos mais importantes para não mudar ou intensificar a terapêutica farmacológica}

\begin{tabular}{|c|c|c|c|c|c|}
\hline \multirow{2}{*}{\multicolumn{2}{|c|}{ Na minha experiência não mudo ou intensifico a terapêutica anti-hipertensora quando o doente... }} & $1 .^{\circ}$ & $2 .^{\circ}$ & 3. ${ }^{\circ}$ & \multirow[t]{2}{*}{ Total } \\
\hline & & \multicolumn{3}{|c|}{ n (\%) } & \\
\hline a. & $\begin{array}{l}\text {... não tem tomado a medicação como indicado, omite tomas ou deixou terminar } \\
\text { a medicação. }\end{array}$ & $\begin{array}{c}6 \\
(10,0)\end{array}$ & $\begin{array}{c}4 \\
(6,7)\end{array}$ & $\begin{array}{c}4 \\
(6,7)\end{array}$ & $\begin{array}{c}14 \\
(23,3)\end{array}$ \\
\hline b. & $\begin{array}{l}\text {... precisa de ser avaliado numa segunda consulta: esta foi a primeira em que a PA está } \\
\text { aumentada. }\end{array}$ & $\begin{array}{c}4 \\
(6,7)\end{array}$ & $\begin{array}{c}5 \\
(8,3)\end{array}$ & $\begin{array}{c}5 \\
(8,3)\end{array}$ & $\begin{array}{c}14 \\
(23,3)\end{array}$ \\
\hline c. & ... tem os valores de PA em auto-monitorização normais. & $\begin{array}{c}6 \\
(10,0)\end{array}$ & $\begin{array}{c}4 \\
(6,7)\end{array}$ & $\begin{array}{c}1 \\
(1,7)\end{array}$ & $\begin{array}{c}11 \\
(18,3)\end{array}$ \\
\hline d. & ... tem HTA de bata branca (a PA só está elevada nesta consulta). & $\begin{array}{c}2 \\
(3,3)\end{array}$ & $\begin{array}{c}1 \\
(1,7)\end{array}$ & $\begin{array}{c}5 \\
(8,3)\end{array}$ & $\begin{array}{c}8 \\
(13,3)\end{array}$ \\
\hline e. & ... não consegue comprar a medicação. & $\begin{array}{c}4 \\
(6,7)\end{array}$ & - & $\begin{array}{c}3 \\
(5,0)\end{array}$ & $\begin{array}{c}7 \\
(11,7)\end{array}$ \\
\hline f. & $\begin{array}{l}\text {... não tem tomado a medicação como indicado porque não compreendeu as minhas } \\
\text { recomendações anteriores. }\end{array}$ & $\begin{array}{c}2 \\
(3,3)\end{array}$ & $\begin{array}{c}2 \\
(3,3)\end{array}$ & $\begin{array}{c}1 \\
(1,7)\end{array}$ & $\begin{array}{c}5 \\
(8,3)\end{array}$ \\
\hline g. & $\begin{array}{l}\text {... não tem um estilo de vida saudável - consumo elevado de sal, álcool em excesso, com } \\
\text { peso elevado ou fumador. }\end{array}$ & $\begin{array}{c}1 \\
(1,7)\end{array}$ & $\begin{array}{c}3 \\
(5,0)\end{array}$ & - & $\begin{array}{c}4 \\
(6,7)\end{array}$ \\
\hline h. & ... está sob stress, irritado ou com dor aguda. & - & $\begin{array}{c}3 \\
(5,0)\end{array}$ & $\begin{array}{c}1 \\
(1,7)\end{array}$ & $\begin{array}{c}4 \\
(6,7)\end{array}$ \\
\hline i. & ... é resistente à ideia de adicionar mais fármacos ou mudar o regime terapêutico. & - & $\begin{array}{c}1 \\
(1,7)\end{array}$ & $\begin{array}{c}1 \\
(1,7)\end{array}$ & $\begin{array}{c}2 \\
(3,3)\end{array}$ \\
\hline j. & ... tem HTA ligeira. & - & - & $\begin{array}{c}2 \\
(3,3)\end{array}$ & $\begin{array}{c}2 \\
(3,3)\end{array}$ \\
\hline k. & ... teve um episódio isquémico recente / Acho adequado manter a PA elevada neste doente. & $\begin{array}{c}1 \\
(1,7)\end{array}$ & - & - & $\begin{array}{c}1 \\
(1,7)\end{array}$ \\
\hline l. & ... está já no seu melhor nível de controlo, após várias tentativas de ajustar a terapêutica. & - & $\begin{array}{c}1 \\
(1,7)\end{array}$ & - & $\begin{array}{c}1 \\
(1,7)\end{array}$ \\
\hline m. & $\begin{array}{l}\text {... é intolerante aos efeitos adversos dos anti-hipertensores (edema, hipotensão ortostática, } \\
\text { fadiga, ...). }\end{array}$ & - & $\begin{array}{c}1 \\
(1,7)\end{array}$ & - & $\begin{array}{c}1 \\
(1,7)\end{array}$ \\
\hline n. & ... tem a HTA diastólica dentro dos valores normais. & - & $\begin{array}{c}1 \\
(1,7)\end{array}$ & - & $\begin{array}{c}1 \\
(1,7)\end{array}$ \\
\hline o. & ... pode vir a ter interacções entre os medicamentos. & - & - & $\begin{array}{c}1 \\
(1,7)\end{array}$ & $\begin{array}{c}1 \\
(1,7)\end{array}$ \\
\hline p. & $\begin{array}{l}\text {... acabou de fumar ou beber café ou outra bebida com cafeína antes desta consulta e por } \\
\text { isso prefiro não considerar esta medição. }\end{array}$ & - & - & $\begin{array}{c}1 \\
(1,7)\end{array}$ & $\begin{array}{c}1 \\
(1,7)\end{array}$ \\
\hline
\end{tabular}

Nota: A pergunta pedia que fossem assinalados os três principais motivos para mudança na terapêutica; na tabela, os motivos estão ordenados pela frequência com que foram assinalados e são indicadas as proporções de médicos que assinalaram como $1 .{ }^{\circ}, 2^{\circ}$ ou $3 .{ }^{\circ}$ principal motivo, para cada opção. HTA - Hipertensão; PA - Pressão arterial; onde indicado "-" considerar "0\%".

petências do doente é importante haver uma maior discussão sobre a terapêutica e sobre os critérios considerados para a decisão clínica. ${ }^{9,13,16}$
A comunicação com o médico é também importante para aumentar o benefício da auto-monitorização da PA. Uma meta-análise recente demonstrou que esta 


\begin{tabular}{|c|c|c|c|c|c|}
\hline & & $1 .^{\circ}$ & $2 .^{\circ}$ & 3. ${ }^{\circ}$ & Total \\
\hline \multicolumn{2}{|r|}{ Na minha experiência, os três principais factores associados à não adesão à terapêutica são: } & \multicolumn{4}{|c|}{ n (\%) } \\
\hline a. & Custos da medicação. & $\begin{array}{c}8 \\
(13,3)\end{array}$ & $\begin{array}{c}4 \\
(6,7)\end{array}$ & $\begin{array}{c}8 \\
(13,3)\end{array}$ & $\begin{array}{c}20 \\
(33,3)\end{array}$ \\
\hline b. & Desinteresse sobre a HTA. & $\begin{array}{c}7 \\
(11,7)\end{array}$ & $\begin{array}{c}5 \\
(8,3) \\
\end{array}$ & $\begin{array}{c}1 \\
(1,7)\end{array}$ & $\begin{array}{c}13 \\
(21,7)\end{array}$ \\
\hline c. & Polimedicação associada a co-morbilidades. & $\begin{array}{c}1 \\
(1,7)\end{array}$ & $\begin{array}{c}5 \\
(8,3)\end{array}$ & $\begin{array}{c}5 \\
(8,3)\end{array}$ & $\begin{array}{c}11 \\
(18,3)\end{array}$ \\
\hline d. & Dificuldade em compreender o seu estado de saúde em geral. & $\begin{array}{c}5 \\
(8,3)\end{array}$ & $\begin{array}{c}4 \\
(6,7)\end{array}$ & $\begin{array}{c}1 \\
(1,7)\end{array}$ & $\begin{array}{c}10 \\
(16,7)\end{array}$ \\
\hline e. & Falta de informação ou informação errada sobre a HTA. & $\begin{array}{c}3 \\
(5,0)\end{array}$ & $\begin{array}{c}3 \\
(5,0)\end{array}$ & $\begin{array}{c}2 \\
(3,3)\end{array}$ & $\begin{array}{c}8 \\
(13,3) \\
\end{array}$ \\
\hline f. & Experiência prévia de efeitos adversos à medicação. & $\begin{array}{c}1 \\
(1,7)\end{array}$ & $\begin{array}{c}1 \\
(1,7)\end{array}$ & $\begin{array}{c}4 \\
(6,7)\end{array}$ & $\begin{array}{c}6 \\
(10,0)\end{array}$ \\
\hline g. & Falta de suporte familiar ou social. & $\begin{array}{c}2 \\
(3,3)\end{array}$ & $\begin{array}{c}2 \\
(3,3)\end{array}$ & $\begin{array}{c}2 \\
(3,3)\end{array}$ & $\begin{array}{c}6 \\
(10,0) \\
\end{array}$ \\
\hline h. & Nível educacional do doente. & - & $\begin{array}{c}1 \\
(1,7)\end{array}$ & $\begin{array}{c}3 \\
(5,0)\end{array}$ & $\begin{array}{c}4 \\
(6,7)\end{array}$ \\
\hline i. & Falta de informação ou informação errada sobre a medicação. & $\begin{array}{c}1 \\
(1,7)\end{array}$ & - & - & $\begin{array}{c}1 \\
(1,7) \\
\end{array}$ \\
\hline j. & Relação com o médico. & - & $\begin{array}{c}1 \\
(1,7) \\
\end{array}$ & - & $\begin{array}{c}1 \\
(1,7) \\
\end{array}$ \\
\hline k. & Regime anti-hipertensor com posologia complexa. & - & $\begin{array}{c}1 \\
(1,7) \\
\end{array}$ & - & $\begin{array}{c}1 \\
(1,7) \\
\end{array}$ \\
\hline l. & Idade do doente. & - & - & $\begin{array}{c}1 \\
(1,7)\end{array}$ & $\begin{array}{c}1 \\
(1,7)\end{array}$ \\
\hline m. & Outro motivo. & $\begin{array}{c}2 \\
(3,3)\end{array}$ & - & - & $\begin{array}{c}2 \\
(3,3)\end{array}$ \\
\hline
\end{tabular}

Nota: A pergunta pedia que fossem assinalados os três principais factores associados à não adesão; na tabela, os factores estão ordenados pela frequência com que foram assinalados e são indicadas as proporções de médicos que assinalaram como $1 .^{\circ}, 2 .^{\circ}$ ou $3 .^{\circ}$ principal factor, para cada opção. HTA - Hipertensão; onde indicado “-” considerar “0 (0,0\%)".

prática permite uma redução de 3,9mmHg na PA sistólica e, em combinação com cuidados de saúde adicionais, uma diminuição entre 3,4 e 8,9mmHg. ${ }^{21}$ Por outro lado, um estudo qualitativo no Reino Unido, com a participação de indivíduos com dispositivos de medição da $\mathrm{PA}$, revelou que um maior envolvimento dos médicos ajudaria a ultrapassar dificuldades na obtenção e interpretação dos valores tensionais..$^{22}$ No presente estu- do, a auto-monitorização da PA foi considerada importante na medida em que ajuda o doente a compreender a sua doença e a participar de forma activa no controlo da PA. Contudo, a maioria dos médicos parece confiar mais nos valores de PA obtidos na consulta, um resultado também descrito noutro estudo. ${ }^{23} \mathrm{~A}$ falta de confiança em alguns dispositivos utilizados (e.g., de pulso) e na medição adequada são alguns dos factores 
que podem justificar a preferência pelos valores registados na consulta. ${ }^{23-25}$

O diagnóstico da HTA requer a medição da PA, que pode ser realizada no contexto da consulta como, em algumas situações, com recurso à monitorização em ambulatório (MAPA). ${ }^{6,26}$ Neste estudo, a média dos valores considerados aceitáveis na medição da PA por MAPA são ligeiramente discordantes daqueles que definem hipertensão no período diurno ( $\geq$ 135/85 $\mathrm{mmHg}) .{ }^{6}$ Apesar da reconhecida importância da MAPA no diagnóstico e prognóstico de complicações cardiovasculares (principalmente a MAPA no período nocturno) ${ }^{6,27}$ não estão ainda definidos os valores alvo para a definição de HTA tratada e controlada, sendo que a inclusão da MAPA na avaliação de rotina de um hipertenso tem custos para o sistema e para o doente. ${ }^{28-29}$

A adopção de estilos de vida saudáveis é outro determinante da redução do risco cardiovascular e controlo tensional. ${ }^{6}$ Todos os médicos consideraram as modificações do estilo de vida úteis para o controlo da PA elevada, recomendando-as, apesar de reconhecerem que a sua implementação é difícil, principalmente nos idosos com mais de 75 anos. As medidas não farmacológicas escolhidas pelos médicos estão de acordo com as normas de orientação clínica, ${ }^{6,30}$ sendo recomendadas a partir de valores de PA sistólica/diastólica de 140/90mmHg num indivíduo sem outros factores de risco. Contudo, os médicos consideram que apenas $7 \%$ dos seus doentes se encontram controlados apenas com medidas não farmacológicas, o que pode estar relacionado com a falta de adesão a longo prazo e a grande variabilidade de resposta dos valores de PA a estas medidas. ${ }^{6}$ Conhecer os estilos de vida do doente e aconselhar a modificação destes parecem ser componentes frequentemente realizadas pelos médicos participantes. Outros estudos reportam que as actividades preventivas e de promoção da saúde são realizadas de uma forma genérica (não adaptada à realidade de cada utente) e com menor frequência comparativamente a outras actividades, devido ao volume de trabalho, falta de tempo e de incentivos. ${ }^{31-32}$

Os valores de PA considerados para a decisão de iniciar a terapêutica farmacológica e como alvo a atingir têm sido revistos na última década em diferentes recomendações. Em 2007, as recomendações da Sociedade Europeia de Hipertensão indicavam como alvo manter a PA $<140 / 90 \mathrm{mmHg}$ em hipertensos sem outros factores de risco (incluindo idosos) e $<130 / 80 \mathrm{mmHg}$ em in divíduos com diabetes mellitus ou risco cardiovascular elevado associado à existência de doença cardiovascular ou renal. ${ }^{14}$ Contudo, estas recomendações foram revistas em 2009, reconhecendo a necessidade de mais evidência no suporte aos valores a considerar para os hipertensos idosos e diabéticos/doentes renais. ${ }^{15} \mathrm{Em}$ 2013, com a divulgação de outros estudos, as recomendações passaram a indicar como alvo manter a PA $<140 / 90 \mathrm{mmHg}$ em hipertensos com ou sem factores de risco (nos diabéticos $<140 / 85 \mathrm{mmHg}$ ) e, nos indivíduos com mais de 80 anos, manter a PA entre 140-150 / < 90mmHg (Quadro VII). ${ }^{6}$ Outras recomendações, como as do JNC 8, NICE e recomendações Canadianas, também indicam valores mais elevados no idoso, apesar de existirem algumas inconsistências na definição da faixa etária. ${ }^{7,26,33}$

No nosso estudo verificou-se que a maioria dos médicos decide iniciar medicação anti-hipertensora acima do valor recomendado à data do estudo. ${ }^{15}$ De notar que, em hipertensos com diabetes mellitus ou insuficiência renal, a maioria dos médicos decide iniciar terapêutica a partir de valores mais baixos - PA sistólica/diastólica de 130/80mmHg, o que está de acordo com essas mesmas recomendações. ${ }^{15}$ Contudo, para hipertensos com insuficiência cardíaca, a maioria dos médicos considerou os mesmos valores da população sem outros factores de risco cardiovascular, sendo recomendados na altura valores mais baixos $(130 / 80 \mathrm{mmHg})$ para hipertensos com doença cardiovascular. ${ }^{15}$ No contexto de um episódio agudo de insuficiência cardíaca, se o doente apresentar PA baixa, normal ou elevada reflecte diferentes mecanismos fisiopatológicos e consequentes respostas ao tratamento, tendo sido observado que valores de PA mais elevados na admissão hospitalar são preditores de maior sobrevivência dos doentes com insuficiência cardíaca. ${ }^{34}$ Como não existe evidência de benefício acrescido em iniciar e manter terapêutica anti-hipertensora com valores de PA inferiores, as recomendações mais actuais indicam os valores 140/90mmHg em geral como objectivo a atingir também neste grupo de hipertensos, como para os diabéticos e hipertensos com insuficiência renal. ${ }^{6,26,30}$

As normas clínicas de 2013 também trouxeram a 


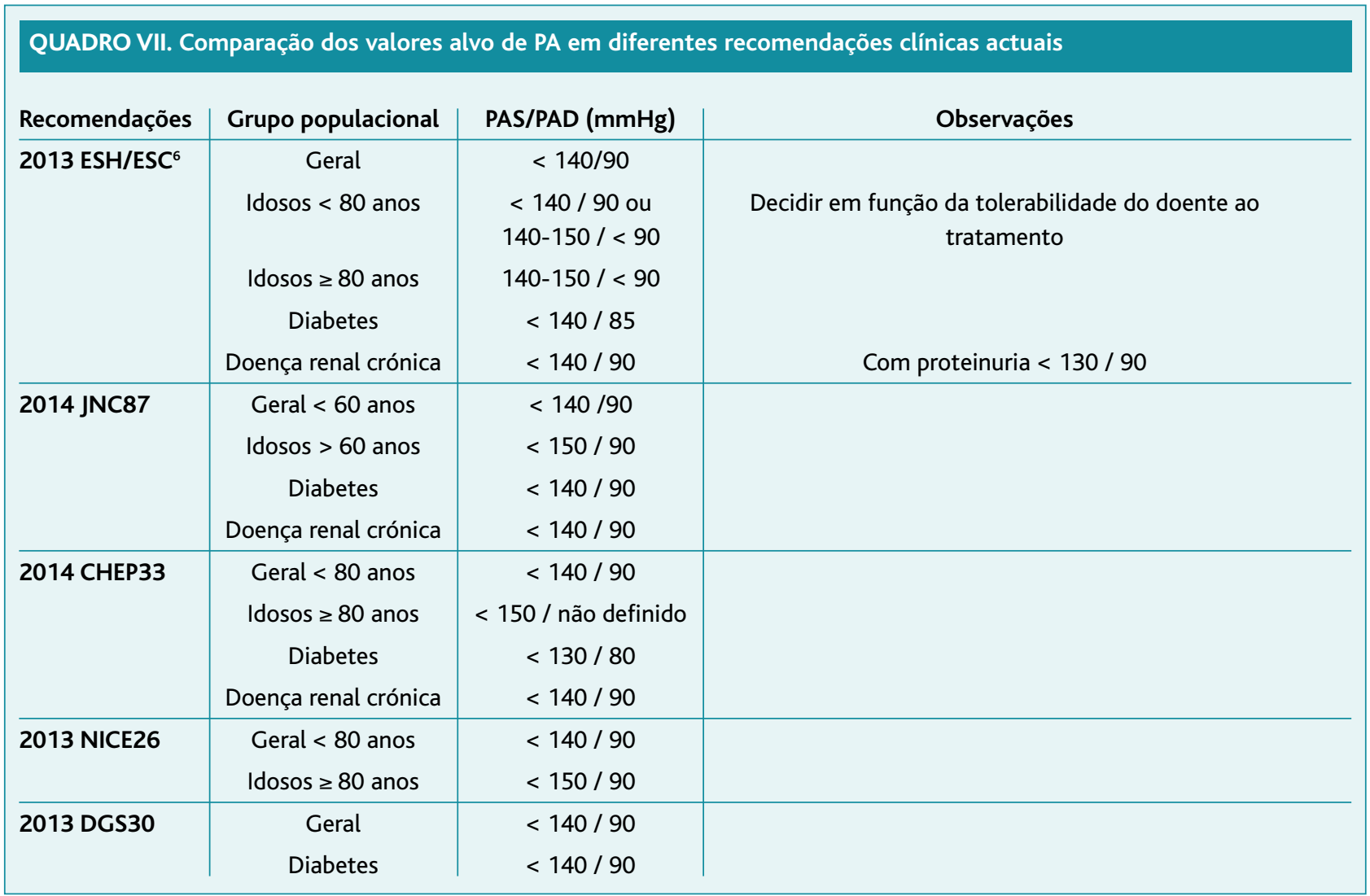

Nota: ESH - European Society of Hypertension; ESC - European Society of Cardiology; JNC - Joint National Committee; CHEP - Canadian Health Education Program; NICE - National Institute for Health and Care Excellence; DGS - Direcção-Geral da Saúde.

revisão dos valores-alvo de PA no idoso, sendo agora recomendado que a PA sistólica se mantenha entre 140 a $150 \mathrm{mmHg}$ em indivíduos com menos de 80 anos e que, em hipertensos com mais de 80 anos, a redução para estes valores deve considerar o estado físico e cognitivo do doente. ${ }^{6-7}$ No presente estudo, a maioria dos médicos concordava serem aceitáveis valores de PA mais elevados nos idosos. Apesar das recomendações anteriores preconizarem o início da medicação em função do risco cardiovascular global e independentemente da idade, ${ }^{14-15}$ os médicos participantes indicaram iniciar terapêutica para PA sistólica/diastólica a partir de 150/90 mmHg, à semelhança de outros estudos. ${ }^{19}$ As questões de tolerabilidade à redução da PA e à medicação são importantes na definição sobre qual o valor ideal de PA sistólica a atingir, sendo que a maioria dos médicos concordava que haveria mais idosos do que in- divíduos mais jovens a terem de descontinuar a terapêutica devido aos efeitos secundários. ${ }^{6}$

Neste estudo, os valores considerados para uma HTA controlada foram inferiores aos propostos pelas recomendações clínicas de 2007 e 2009..$^{14-15,17}$ Contudo, os valores reportados para doentes com menor risco cardiovascular são próximos dos observados num estudo europeu, onde os valores médios com os quais os médicos participantes indicaram sentir-se satisfeitos eram de 131,6 / 81,9mmHg, passando a ficar mais atentos quando os valores passavam a 148,9 / 91,6mmHg. ${ }^{35}$

Quanto à decisão sobre a medicação anti-hipertensora, os médicos parecem preferir iniciar terapêutica em monoterapia, independentemente das características do hipertenso. No entanto, consideraram que, no ano anterior ao estudo, apenas $27 \%$ dos seus doentes se encontravam controlados em monoterapia. Esta 
proporção vai ao encontro dos resultados de outros estudos que têm demonstrado que a monoterapia com qualquer classe de anti-hipertensores só possibilita o adequado controlo tensional em menos de $20-30 \%$ da população hipertensa, exceptuando-se os doentes com HTA grau $1 .{ }^{36}$ Desta forma, as recomendações clínicas mais recentes sugerem, nos doentes com risco cardiovascular elevado, o início da terapêutica anti-hipertensora com dois fármacos em dose baixa. ${ }^{6-7,30}$

Num indivíduo sem outros factores de risco, a maioria dos médicos indicou preferir iniciar medicação anti-hipertensora com, por ordem decrescente, IECAs, ARAs, tiazidas, BB, diuréticos não tiazídicos e ACCs. Estes resultados são diferentes dos encontrados por Cortez-Dias e colaboradores - diuréticos (47\%), ARAs (43\%), IECAs (39\%), ACCs (19\%) e BB (16\%) - mas semelhantes aos descritos num estudo com a rede Médicos-Sentinela. ${ }^{36-37}$ As recomendações mais recentes são no sentido de que pode ser usado qualquer fármaco de $1^{\text {a }}$ linha em monoterapia, uma vez que as principais classes não diferem significativamente na sua capacidade de reduzir a PA. Contudo, em situações de lesão de órgão-alvo e de protecção da função renal pode haver vantagem no bloqueio do eixo renina-angiotensina-aldosterona. ${ }^{26,30}$

O termo "inércia clínica" tem sido utilizado para descrever a ausência de intensificação terapêutica num doente que não tenha ainda atingido os objectivos, isto é, que apresente ainda valores de PA superiores aos recomendados. ${ }^{1,35}$ Contudo, é importante distinguir entre potencial inércia clínica e ausência de intensificação da terapêutica como decisão adequada ao indivíduo em particular. ${ }^{20,38}$ Neste estudo, os motivos mais indicados para não mudar ou intensificar a terapêutica se a PA não estiver controlada numa dada consulta foram a não-adesão do doente à terapêutica (também um dos motivos mais indicados para a mudança terapêutica), a necessidade de uma segunda avaliação que confirme os valores tensionais elevados e o verificar que os valores em auto-monitorização se encontram normais. Estes motivos parecem enquadrar-se numa inércia adequada e não no conceito de inércia clínica, tendo sido descritos também noutros estudos. ${ }^{20,39}$

A não adesão é um problema frequente na terapêutica anti-hipertensora como em outras terapêuticas crónicas. ${ }^{40}$ Neste estudo, os médicos consideraram que cerca de um terço dos seus doentes seriam não-aderentes. Os custos da medicação, o desinteresse do doente quanto à sua HTA e a polimedicação foram apontados como os principais factores associados a este problema. Chin e colaboradores reportaram uma proporção mais elevada de não-adesão (79\%), dos quais 51\% indicaram razões financeiras que, ao não serem abordadas com o médico de família, impossibilitam a mudança terapêutica e o controlo da HTA. ${ }^{41}$

Estudos sobre a prática médica são susceptíveis a várias limitações. ${ }^{35}$ Uma das limitações deste estudo consiste no ser baseado em informação fornecida pelo médico e não na observação directa. Nesse sentido, não é possível excluir a possibilidade de viés no sentido de adaptar as respostas ao que se julga ser a prática clínica óptima. A selecção da amostra poderá também influenciar os resultados observados, na medida em que participaram médicos que já tinham aceitado participar no estudo DIMATCH-HTA, um estudo observacional sobre HTA, e que poderiam estar mais informados e interessados sobre a doença e recomendações. Além disso, o próprio desenho do estudo DIMATCH-HTA limitou a selecção de unidades de saúde à região de Lisboa e Vale do Tejo, limitando a generalização dos resultados para outras regiões. Por último, apesar de a dimensão da amostra ser superior à participação portuguesa em estudos semelhantes, ${ }^{35}$ é ainda inferior à necessária para uma potência estatística adequada na identificação de associações com características dos médicos menos frequentes, não permitindo a realização de uma análise multivariada. Um estudo nacional sobre este tema poderia trazer mais informação, como o grau de acordo com e implementação das recomendações europeias e nacionais mais recentes sobre risco cardiovascular.

\section{CONCLUSÃO}

Em relação à atitude dos médicos no controlo da HTA, face às recomendações e normas clínicas à altura do estudo, verificou-se:

- o reconhecimento da utilidade da modificação de estilos de vida;

- alguma heterogeneidade relativamente aos critérios de diagnóstico da MAPA;

- a preferência pelos valores de pressão arterial registados na consulta; 
- alguma divergência quanto aos alvos terapêuticos nos idosos;

- a preferência pelo início da terapêutica em monoterapia.

A divulgação de nova evidência leva à necessidade de actualizar regularmente as recomendações para o controlo da HTA. Este processo requer, da parte do médico de família, um investimento na sua formação contínua de forma a incorporar na sua prática clínica as melhores opções para o diagnóstico e seguimento dos seus hipertensos. No entanto, a promoção da saúde ao nível dos cuidados de saúde primários depende do desenvolvimento de um modelo de participação mútua, onde o doente é chamado a ter um papel activo na decisão terapêutica. Assim, o médico de família está numa posição privilegiada para promover a comunicação com o doente e, com isso, capacitá-lo para a gestão da doença e promover uma melhor adesão à terapêutica.

\section{AGRADECIMENTOS}

Os autores agradecem aos médicos e USF/USCP participantes no estudo e aos respectivos Agrupamentos de Centros de Saúde (ACES) que aceitaram colaborar:

- ACES Oeiras: USF Dafundo (estudo piloto)

- ACES Lisboa Norte: Centro de Saúde de Alvalade e USF Tílias (estudo piloto)

- ACES Loures: Centro de Saúde de Sacavém (ext. Sacavém A, Moscavide e Camarate) e USF São João da Talha

- ACES Amadora: Centro de Saúde da Amadora e USF Arco-Íris

- ACES Algueirão-Rio de Mouro: Centro de Saúde do Algueirão e USF da Natividade

- ACES Cacém-Queluz: Centro de Saúde Queluz e USF Mãe de Água

Agradecem ainda à equipa de entrevistadores do projecto DIMATCHHTA e a Rui Simões pelos comentários adicionais.

\section{REFERÊNCIAS BIBLIOGRÁFICAS}

1. Nicodème R,Albessard A,Amar J, Chamontin B, Lang T. Poor blood pressure control in general practice: in search of explanations. Arch Cardiovasc Dis. 2009;102(6-7):477-83.

2. Alwan A. Global status report on noncommunicable diseases 2010. Reprinted ed. Geneva: World Health Organization; 2011. ISBN 9789240686458

3. Bromfield S, Muntner P. High blood pressure: the leading global burden of disease risk factor and the need for worldwide prevention programs. Curr Hypertens Rep. 2013;15(3):134-6.

4. Polonia J, Martins L, Pinto F, Nazare J. Prevalence, awareness, treatment and control of hypertension and salt intake in Portugal: changes over a decade. J Hypertens. 2014;32(6):1211-21.

5. World Health Organization. A global brief on hypertension: silent kil- ler, global public health crisis - World Health Day 2013. Geneva:WHO; 2013.

6. Mancia G, Fagard R, Narkiewicz K, Redon J, Zanchetti A, Böhm M, et al. 2013 ESH/ESC guidelines for the management of arterial hypertension: the Task Force for the Management of Arterial Hypertension of the European Society of Hypertension (ESH) and of the European Society of Cardiology (ESC). Eur Heart J. 2013;34(28):2159-219.

7. James PA, Oparil S, Carter BL, Cushman WC, Dennison-Himmelfarb C, Handler J, et al. 2014 evidence-based guideline for the management of high blood pressure in adults: report from the panel members appointed to the Eighth Joint National Committee (JNC 8). JAMA. 2014;311(5):507-20

8. Cabana MD, Rand CS, Powe NR, Wu AW, Wilson MH, Abboud PA, et al. Why don't physicians follow clinical practice guidelines? A framework for improvement. JAMA. 1999;282(15):1458-65.

9. Makoul G, Arntson P, Schofield T. Health promotion in primary care: physician-patient communication and decision making about prescription medications. Soc Sci Med. 1995;41(9):1241-54.

10. Rodrigues JG. Porque consultam os utentes o seu médico de família? [Why do patients consult their family doctor?]. Rev Port Clin Geral. 2000;16(6):442-52. Portuguese

11. Sharma AM, Wittchen HU, Kirch W, Pittrow D, Ritz E, Göke B, et al. High prevalence and poor control of hypertension in primary care: cross-sectional study. J Hypertens. 2004;22(3):479-86.

12. Brotons C, Bulc M, Sammut MR, Sheehan M, Manuel da Silva Martins $C, B j o ̈ r k e l u n d ~ C$, et al. Attitudes toward preventive services and lifestyle: the views of primary care patients in Europe - The EUROPREVIEW patient study. Fam Pract. 2012;29 Suppl 1:i168-76.

13. WONCA Europe. The European definition of general practice/family medicine. London:WONCA Europe; 2002.

14. Mancia G, De Backer G, Dominiczak A, Cifkova R, Fagard R, Germano $\mathrm{G}$, et al. 2007 ESH-ESC Practice guidelines for the management of arterial hypertension: ESH-ESC Task Force on the Management of Arterial Hypertension. J Hypertens. 2007;25(9):1751-62.

15. Mancia G, Laurent S, Agabiti-Rosei E, Ambrosioni E, Burnier M, Caulfield MJ, et al. Reappraisal of European guidelines on hypertension management: a European Society of Hypertension Task Force document. J Hypertens. 2009;27(11):2121-58.

16. Chobanian AV, Bakris GL, Black HR, Cushman WC, Green LA, Izzo Jr JL, et al. Seventh report of the Joint National Committee on prevention, detection, evaluation, and treatment of high blood pressure. Hypertension. 2003;42(6):1206-52.

17. Direcção-Geral da Saúde. Diagnóstico, tratamento e controlo da hipertensão arterial: circular normativa n 2/DGCG, de 31/03/2004. Lisboa: DGS; 2004.

18. Polónia J, Ramalhinho V, Martins L, Saavedra J. Normas sobre detecção, avaliação e tratamento da hipertensão arterial da Sociedade Portuguesa de Hipertensão. Rev Port Cardiol. 2006;25(6):649-60.

19. Duggan S, Ford GA, Eccles M. Doctors' attitudes towards the detection and treatment of hypertension in older people. J Hum Hypertens. 1997;11(5):271-6.

20. Safford MM, Shewchuk R, Qu H, Williams JH, Estrada CA, Ovalle F, et al. Reasons for not intensifying medications: differentiating 'clinical inertia' from appropriate care. J Gen Intern Med. 2007;22(12):1648-55. 
21. Uhlig K, Patel K, Ip S, Kitsios GD, Balk EM. Self-measured blood pressure monitoring in the management of hypertension: a systematic review and meta-analysis. Ann Intern Med. 2013;159(3):185-94.

22. Vasileiou K, Barnett J, Young T. Interpreting and acting upon home blood pressure readings: a qualitative study. BMC Fam Pract. 2013;14:97.

23. Logan AG, Dunai A, Mclsaac WJ, Irvine MJ, Tisler A. Attitudes of primary care physicians and their patients about home blood pressure monitoring in Ontario. J Hypertens. 2008;26(3):446-52.

24. Tislér A, Dunai A, Keszei A, Fekete B, Othmane Tel H, Torzsa P, et al. Primary-care physicians' views about the use of home/self blood pressure monitoring: nationwide survey in Hungary. J Hypertens. 2006;24(9): 1729-35.

25. McManus RJ, Wood S, Bray EP, Glasziou P, Hayen A, Heneghan C, et al. Self-monitoring in hypertension: a web-based survey of primary care physicians. J Hum Hypertens. 2014;28(2):123-7.

26. National Clinical Guideline Centre. Hypertension: clinical management of primary hypertension in adults: update of clinical guidelines 18 and 34. London: London College of Physicians; 2011.

27. O'Brien E, Asmar R, Beilin L, Imai Y, Mancia G, Mengden T, et al. Practice guidelines of the European Society of Hypertension for clinic, ambulatory and self blood pressure measurement. J Hypertens. 2005;23 (4):697-701.

28. Head GA, Mihailidou AS, Duggan KA, Beilin LJ, Berry N, Brown MA, et al. Definition of ambulatory blood pressure targets for diagnosis and treatment of hypertension in relation to clinic blood pressure: prospective cohort study. BMJ. 2010;340:c1104.

29. Aguiar H, Silva Al, Pinto F, Catarino S. Avaliação da pressão arterial no ambulatório: revisão baseada na evidência [Ambulatory evaluation of blood pressure: an evidence-based review]. Rev Port Clin Geral. 2011;27(4):362-76. Portuguese

30. Direcção-Geral da Saúde. Abordagem terapêutica da hipertensão arterial: norma da DGS n026/2011, de 29/09/2011. Lisboa: DGS; 2013.

31. Brotons $C$, Björkelund C, Bulc M, Ciurana R, Godycki-Cwirko M, Jurgova $E$, et al. Prevention and health promotion in clinical practice: the views of general practitioners in Europe. Prev Med. 2005;40(5):595-601.

32. Pinto D, Corte-Real S, Nunes JM. Actividades preventivas e indicadores: quanto tempo sobra? [Preventive activities and performance indicators: how much time is left?] Rev Port Clin Geral. 2010;26(5):45564. Portuguese

33. Dasgupta K, Quinn RR, Zarnke KB, Rabi DM, Ravani P, Daskalopoulou SS, et al. The 2014 Canadian Hypertension Education Program recommendations for blood pressure measurement, diagnosis, assessment of risk, prevention, and treatment of hypertension. Can J Cardiol. 2014;30 (5):485-501.

34. Pang PS, Komajda M, Gheorghiade M. The current and future management of acute heart failure syndromes. Eur Heart J. 2010;31(7):78493.

35. Redon J, Erdine S, Böhm M, Ferri C, Kolloch R, Kreutz R, et al. Physician attitudes to blood pressure control: findings from the Supporting Hy- pertension Awareness and Research Europe-wide survey. J Hypertens. 2011;29(8):1633-40.

36. Cortez-Dias N, Martins S, Belo A, Fiuza M. Prevalência e padrões de tratamento da hipertensão arterial nos cuidados de saúde primários em Portugal: resultados do Estudo Valsim [Prevalence and management of hypertension in primary care in Portugal: insights from the VALSIM study]. Rev Port Cardiol. 2009;28(5):499-523. Portuguese

37. Souto D, Simões JA, Torre C, Mendes Z, Marinho Falcão I, Ferreira F, et al. Perfil terapêutico da hipertensão na Rede Médicos Sentinela: 12 anos depois [Prescribing patterns for hypertension in the Portuguese sentinet practice network]. Rev Port Med Geral Fam. 2013;29(5):286-96. Portuguese

38. Fürthauer J, Flamm M, Sönnichsen A. Patient and physician related factors of adherence to evidence based guidelines in diabetes mellitus type 2, cardiovascular disease and prevention: a cross sectional study. BMC Fam Pract. 2013;14:47.

39. Oliveria S, Lapuerta P, McCarthy BD, L'Italien GJ, Berlowitz DR, Asch SM. Physician-related barriers to the effective management of uncontrolled hypertension. Arch Intern Med. 2002;162(4):413-20.

40. Bokhour BG, Berlowitz DR, Long JA, Kressin NR. How do providers assess antihypertensive medication adherence in medical encounters? J Gen Intern Med. 2006;21(6):577-83.

41. Chin A, Alves M, Martins N, Ferreira A, Barbeiro C, Mota M. Influência dos factores financeiros no cumprimento da medicação [Influence of financial factors on adherence to medication]. Rev Port Med Geral Fam. 2012;28(5):368-74. Portuguese

\section{FINANCIAMENTO DO ESTUDO}

O estudo DIMATCH-HTA foi financiado pela Fundação para a Ciência e Tecnologia, com a bolsa PTDC/SAL ESA/103511/2008, pelos Laboratórios Delta - Rottapharm|Madaus e foi premiado pela Fundação Astrazeneca.

\section{CONFLITO DE INTERESSES}

Os autores declaram não ter conflitos de interesses.

\section{COMISSÃO DE ÉTICA}

Estudo realizado após parecer favorável da Comissão de Ética da Faculdade de Medicina da Universidade de Lisboa.

\section{ENDEREÇO PARA CORRESPONDÊNCIA}

Milene Fernandes

Instituto de Medicina Preventiva e Saúde Pública

Faculdade de Medicina de Lisboa

Av. Prof. Egas Moniz - Ed. Egas Moniz, 1649-028 Lisboa.

Telefone: +351 217999422. Fax: +351217999421

Email: mccf@medicina.ulisboa.pt

Recebido em 24-07-2014

Aceite para publicação em 19-05-2015 


\section{ABSTRACT}

\section{CLINICAL DECISION MAKING IN HYPERTENSION: A SURVEY OF ATTITUDES OF FAMILY PHYSICIANS IN THE LISBON HEALTH REGION}

Objectives: This study was designed to characterize the attitudes of family physicians regarding clinical decision making in hypertension, including issues related to monitoring and treatment and to compare these attitudes with clinical recommendations available at the time of the study.

Type of study: Cross-sectional study.

Setting: Primary health care centers in Lisbon and Vale do Tejo, Portugal.

Population: Family physician specialists and trainees in family medicine.

Methods: Self-completed questionnaire assessing variables related to demographic characteristics, experience of treating hypertensive patients, measurement of blood pressure (BP), lifestyle changes, the decision to initiate antihypertensive drugs, and strategies used when assessing patient adherence. Bivariate analysis (Chi-square tests, $\alpha=0.05$ ) was used to explore the associations between clinical decision-making and other variables.

Results: The 60 participants (41 females) from 14 Primary Health Care Centers had a mean age of $52 \pm 8.6$ years and a mean of $22 \pm 8.2$ years experience in practice. All physicians considered lifestyle change to be useful. Acceptable ambulatory BP (ABPM) was $136 / 83 \mathrm{mmHg}$ for the daytime value. The BP values chosen for initiation of treatment were $140 / 90 \mathrm{mmHg}$ for patients without other risk factors. In the elderly, $77 \%$ of physicians agreed with accepting higher BP values, initiating treatment when BP $>150 / 90 \mathrm{mmHg}$. Physicians preferred to initiate therapy with only one drug. Most physicians (58\%) believed that many patients can achieve control of BP with only one drug and physicians with over 20 years of experience in practice tended to agree more with this statement $(p=0.043)$.

Conclusions: The reported attitudes were in line with the national and European clinical guidelines available at the time of the study regarding the usefulness of lifestyle changes and the BP values chosen for initiating treatment. There was some heterogeneity regarding management of BP in the elderly and ABPM values. Updating of clinical recommendations requires continuous training of family physicians, in order to incorporate the best options for management of hypertension in clinical practice.

Keywords: Hypertension; Decision Making; Clinical Guidelines; Physician-patient Relations. 